\title{
The Degree Analysis of an Inhomogeneous Growing Network with Two Types of Vertices
}

\author{
Huilin Huang \\ College of Mathematics and Information Science, Wenzhou University, Zhejiang 325035, China \\ Correspondence should be addressed to Huilin Huang; huilin_huang@sjtu.org
}

Received 11 March 2014; Accepted 27 April 2014; Published 12 May 2014

Academic Editor: Derui Ding

Copyright (C) 2014 Huilin Huang. This is an open access article distributed under the Creative Commons Attribution License, which permits unrestricted use, distribution, and reproduction in any medium, provided the original work is properly cited.

\begin{abstract}
We consider an inhomogeneous growing network with two types of vertices. The degree sequences of two different types of vertices are investigated, respectively. We not only prove that the asymptotical degree distribution of type $s$ for this process is power law with exponent $2+\left((1+\delta) q_{s}+\beta\left(1-q_{s}\right)\right) / \alpha q_{s}$, but also give the strong law of large numbers for degree sequences of two different types of vertices by using a different method instead of Azuma's inequality. Then we determine asymptotically the joint probability distribution of degree for pairs of adjacent vertices with the same type and with different types, respectively.
\end{abstract}

\section{Introduction}

Recently there has been much interest in studying inhomogeneous large-scale networks and attempting to model their topological properties. The classical random graph models are generally homogeneous, in the sense that all the vertices come in the same type (for static random graphs, see [1]; for growing random graphs, see $[2,3])$. In contrast, many large real-world graphs are highly 0 inhomogeneous. In fact, vertices of many real networks are born of difference, and this difference by birth may influence the evolving of the networks to some extent. In order to depict such phenomenon, Söderberg [4] presented a class of inhomogeneous random graph models of order $N$, by means of a straightforward generalization of the classic E-R model to a situation where vertices may come in different types, such that the probability that an edge arises depends on the types of its pair of terminal vertices. Bollobás et al. [5], based on the work of Söderberg [4], introduce a model of an inhomogeneous random graph with conditional independence between the edges; moreover various results have been proved. Their model also includes some special cases such as the CHKNS model [6] and Turova's model [7-10]. Recently, van der Hofstad [11] studies the critical behavior of inhomogeneous random graphs where edges are present independently but with unequal edge occupation probabilities and shows that this critical behavior depends sensitively on the asymptotic properties of their degree sequence. For more substantial details about such inhomogeneous random graph, we can see van der Hofstad [12]. Most of those models are static and do not involve the effect of preferential attachment. Papadopoulos et al. [13] point out that it is very important to study inhomogeneous growing networks which combine the effects of popularity and similarity. There are also many papers studying synchronization control of dynamical networks; see [14] and the references therein.

In this paper, our main purpose is to define an inhomogeneous model which can combine the effects of preferential attachment and difference by birth and then to provide quantitative descriptions of its properties of degree sequence. Our model inherits certain features from homogeneous growing model such that it is capable of producing asymptotic degree distributions such as power law distributions and exponential distributions by choosing proper parameters.

1.1. Definition of Our Model. At first, we introduce a type space $\mathcal{S}=\{0,1\}$. Let $\left\{Y_{t} ; t \geq 2\right\}$ be a sequence of independent random variables with identical distribution (i.i.d. for short), which take values in $\delta$. The distribution of the random variables $\left\{Y_{t} ; t \geq 2\right\}$ is given by

$$
P\left(Y_{t}=0\right)=q_{0}, \quad P\left(Y_{t}=1\right)=q_{1},
$$


where $q_{0}+q_{1}=1$. Let $0<\alpha, \beta<1$, and $\alpha+\beta=1$. Here and thereafter we let

$$
Y_{0} \equiv 0, \quad Y_{1} \equiv 1 .
$$

Now we can define our model based on the information of $\left\{Y_{t} ; t \geq 0\right\}$. Consider the following process which generates a sequence of graphs $\left\{G_{t}\right\}_{t \geq 1}$.

Time-Step 1. Let $G(1)$ be a graph consisting of two vertices $v_{0}$ and $v_{1}$ with one edge connecting them, which are associated by two type variables $Y_{0}$ and $Y_{1}$, respectively.

Time-Step $t \geq 2$. The graph $G(t)$ is constructed from $G(t-1)$ in such a way that a vertex $v_{t}$ associated with one new edge, whose type is controlled by a random variable $Y_{t}$, is added to the graph $G(t-1)$. Denote the degrees of the vertices $\left\{v_{0}, v_{1}, \ldots, v_{t-1}\right\}$ by $d_{0}(r), d_{1}(r), \ldots, d_{t-1}(r)$ at time $r \geq t-1$. For simplicity of the notations, we also denote $d_{i}(r) I_{\left\{Y_{i}=s\right\}}$ by $d_{i}^{(s)}(r)$, and for $i \geq 0$, we write $\delta^{(i, s)}$ for $\delta I_{\left\{Y_{i}=s\right\}}$, where $\delta$ is a nonnegative real number, and $I_{\{\cdot\}}$ is the indicator function. When the new vertex arrives at the system, the endpoint of the new edge emanating from vertex $v_{t}$ is chosen independently from $\left\{v_{0}, v_{1}, \ldots, v_{t-1}\right\}$ according to their different types. follows.

The attachment procedures of the new edge proceeded as

(a) With probability $\alpha$, it is preferential to attach to an old vertex whose type is the same as the new one. When the vertices $v_{i}$ and $v_{t}$ are of the same type $s$, the probability that $v_{i}$ is chosen as the endpoint of the new edge associated with the new vertex $v_{t}$ is equal to $\alpha\left(\left(d_{i}^{(s)}(t-1)+\delta^{(i, s)}\right) / \sum_{\mathrm{j}=0}^{t-1}\left(d_{j}^{(s)}(t-1)+\delta^{(j, s)}\right)\right)$, so that, for $i=0,1, \ldots, t-1$ and $s=0,1$,

$$
\begin{gathered}
\operatorname{Pr}\left(v_{t} \longrightarrow v_{i}, Y_{i}=s, Y_{t}=s \mid \mathscr{F}_{t-1}\right) \\
=\alpha q_{s} \frac{d_{i}^{(s)}(t-1)+\delta^{(i, s)}}{\sum_{j=0}^{t-1}\left(d_{j}^{(s)}(t-1)+\delta^{(j, s)}\right)} .
\end{gathered}
$$

(b) With probability $\beta=1-\alpha$, it is equitable to connect to an old vertex whose type is different from the new one and the probability that $v_{i}$ is chosen is

$$
\begin{aligned}
& \operatorname{Pr}\left(v_{t} \longrightarrow v_{i}, Y_{i}=1-s, Y_{t}=s \mid \mathscr{F}_{t-1}\right) \\
& \quad=\beta q_{s} \frac{I_{\left\{Y_{i}=1-s\right\}}}{\sum_{j=0}^{t-1} I_{\left\{Y_{j}=1-s\right\}}} ;
\end{aligned}
$$

here and thereafter we let $\mathscr{F}_{t}$ be the $\sigma$-algebra associated with the probability space up to time $t$.

Remark 1. In our model, (3) depicts preferential competitive mechanism among the vertices with the same type. On the other hand, (4) describes the fair competitive effect on the vertices which are of different types. In our model, we can consider the degree of a vertex as an indication of its success, so that vertices with large degree correspond to successful vertices. Naturally, in reality, both the previous success of a vertex and its initial type may play important roles in the final success of the vertex. In our model individuals arrive at the network with different types and one initial edge, which form the basis for their future success. Heuristically, the larger the group is, the more the individuals which are successful exist; on the other hand, the rich will be richer as time increases.

Remark 2. Our model is different from the inhomogeneous model [5] in that it is dynamic; more precisely, a new vertex is added to the graph at each integer time. If $\beta=0$ and $q_{0}=1$, that is, $\operatorname{Pr}\left(Y_{t}=0\right)=1$ for all $t \geq 2$, our model reduces to the original preferential model which is very similar to the one from Barabási and Albert [2], once the types are ignored.

1.2. States of Our Main Results. What we are interested in is the limit distribution of the degree sequences of different types in the resulting graph $G(t)$. Let $N_{k}^{(s)}(t)$ be the number of vertices of type $s$ with degree $k$ in graph $G(t)$. Define $P_{k}^{(s)}(t)=$ $N_{k}^{(s)}(t) /(t+1)$ as the fraction of vertices of type $s$ with degree $k$. What we are concerned about is the limiting behavior of $P_{k}^{(s)}(t)$ as $t$ tends to infinity.

Theorem 3. If $0<\alpha<1$, for $k \geq 1$ and $s=0,1$, respectively, one has

$$
\begin{aligned}
& P_{k}^{(s)} \\
& =\lim _{t \rightarrow \infty} \frac{E\left[N_{k}^{(s)}(t)\right]}{t+1} \\
& =q_{s} \times\left(1+\frac{\alpha(1+\delta) q_{s}}{(1+\delta+\alpha) q_{s}+\beta\left(1-q_{s}\right)}+\frac{\beta\left(1-q_{s}\right)}{q_{s}}\right)^{-1} \\
& \cdot \prod_{j=2}^{k}((j-1+\delta \\
& \left.\quad+\frac{\beta\left(1-q_{s}\right)\left[(1+\delta+\alpha) q_{s}+\beta\left(1-q_{s}\right)\right]}{\alpha q_{s}^{2}}\right) \\
& \quad\left(j+\delta+\frac{(1+\delta+\alpha) q_{s}+\beta\left(1-q_{s}\right)}{\alpha q_{s}}\right. \\
& \left.\left.\quad+\frac{\beta\left(1-q_{s}\right)\left[(1+\delta+\alpha) q_{s}+\beta\left(1-q_{s}\right)\right]}{\alpha q_{s}^{2}}\right)^{-1}\right)
\end{aligned}
$$

$$
\begin{gathered}
c_{s}\left(\Gamma\left(k+\delta+\frac{\beta\left(1-q_{s}\right)\left[(1+\delta+\alpha) q_{s}+\beta\left(1-q_{s}\right)\right]}{\alpha q_{s}^{2}}\right)\right. \\
\times\left(\Gamma \left(k+1+\delta+\frac{(1+\delta+\alpha) q_{s}+\beta\left(1-q_{s}\right)}{\alpha q_{s}}\right.\right. \\
\left.\left.\left.+\frac{\beta\left(1-q_{s}\right)\left[(1+\delta+\alpha) q_{s}+\beta\left(1-q_{s}\right)\right]}{\alpha q_{s}^{2}}\right)\right)^{-1}\right)
\end{gathered}
$$

$\approx c_{s} k^{-\left(2+\left((1+\delta) q_{s}+\beta\left(1-q_{s}\right)\right) / \alpha q_{s}\right)}$, 
where $\Gamma(\cdot)$ is the Gamma function and

$c_{s}$

$$
\begin{gathered}
=q_{s} \times\left(1+\frac{\alpha(1+\delta) q_{s}}{(1+\delta+\alpha) q_{s}+\beta\left(1-q_{s}\right)}+\frac{\beta\left(1-q_{s}\right)}{q_{s}}\right)^{-1} \\
\times \Gamma\left(2+\delta+\frac{(1+\delta+\alpha) q_{s}+\beta\left(1-q_{s}\right)}{\alpha q_{s}}\right. \\
\left.+\frac{\beta\left(1-q_{s}\right)\left[(1+\delta+\alpha) q_{s}+\beta\left(1-q_{s}\right)\right]}{\alpha q_{s}^{2}}\right) \\
\times\left(\Gamma\left(1+\delta \quad \frac{\beta\left(1-q_{s}\right)\left[(1+\delta+\alpha) q_{s}+\beta\left(1-q_{s}\right)\right]}{\alpha q_{s}^{2}}\right)\right)^{-1}
\end{gathered}
$$

the empty product, arising when $k=1$, is defined to be equal to one.

Remark 4. (i) If $\alpha=0$, the result graph $G(t)$ is a bipartite graph. Moreover, the degree distributions of different types are as follows: for $k \geq 1$ and $s=0,1$,

$$
P_{k}^{(s)}=\left(1-q_{s}\right)^{k-1} q_{s}^{2} .
$$

(ii) For $\alpha=1$ and $t \geq 1$, the result graph $G(t)$ has two different connected branches such that one branch is composed of the vertices of type 0 , and the vertices of type 1 consist of the other one. Moreover, there is only one initial edge that connects the two branches. Furthermore, for $s=$ 0,1 , the degree distributions of different types of vertices are

$$
P_{k}^{(s)} \approx c_{s}^{\prime} k^{-(3+\delta)},
$$

where $c_{s}^{\prime}$ is equal to the value of $c_{s}$ when $\alpha=1, \beta=0$.

We want to say that the proof of (9) and (10) is very similar to the proof of Theorem 3, so we omitted it.

For all $k \geq 1$, let $N_{k}(t)$ be the number of vertices with degree $k$ in graph $G(t)$. Define $P_{k}(t)=N_{k}(t) /(t+1)$ as the fraction of vertices with degree $k$. Since $N_{k}(t)=N_{k}^{(0)}(t)+$ $N_{k}^{(1)}(t)$, the following corollary is a direct result of Theorem 3 .

Corollary 5. In our model, one has

$$
P_{k}=\lim _{t \rightarrow \infty} \frac{E\left[N_{k}(t)\right]}{t+1}=P_{k}^{(0)}+P_{k}^{(1)},
$$

where $P_{k}^{(s)}$ for $s=0,1$ are defined as in Theorem 3 .

Note the following fact:

$$
\frac{\Gamma(k+a)}{\Gamma(k+b)}=\frac{1}{b-a-1}\left[\frac{\Gamma(k+a)}{\Gamma(k-1+b)}-\frac{\Gamma(k+1+a)}{\Gamma(k+b)}\right] .
$$

By using a telescope sum identity we can deduce the following corollary easily.
Corollary 6. For all $k \geq 1$ and $s=0,1, P_{k}^{(s)}$ are defined by (6); then one has the total fractions of vertices of types 0 and 1 in our limit graph which are equal to $q_{s}(s=0,1)$, respectively; that is,

$$
\sum_{k=1}^{\infty} P_{k}^{(s)}=q_{s}, \quad s=0,1 .
$$

Remark 7. (i) We know that $\left\{Y_{i} ; i \geq 2\right\}$ is a sequence of i.i.d. random variables; thus by strong law of large numbers, $T_{t-1}^{(s)} / t \rightarrow q_{s},(s=0,1)$ as $t \rightarrow \infty$. Thus Corollary 6 is exactly consistent with it.

(ii) From Corollary 5, for $0<\alpha, \beta<1$, we can easily find that if $q_{0}=q_{1}=1 / 2$, the total degree sequence $P_{k}$ follows power law with the exponent $2+(1+\delta+\beta) / \alpha$; otherwise, $P_{k}$ is a linear combination of two different power law distributions.

(iii) From our analysis of the degree distribution of different types, we can find that our model grasps two heuristic phenomena as follows: one is "rich-get-richer" effect; the other is that the larger the group is, the more the successful individuals in that group are.

We are also concerned about the strong law of large numbers of degree sequences for different types, respectively, as follows.

Theorem 8. For $s \in \mathcal{S}$ and fixed $k \geq 1$, one has

$$
\lim _{t \rightarrow \infty} P_{k}^{(s)}(t)=P_{k}^{(s)}, \quad \text { a.s. }
$$

At last, it is also interesting to find out an expression for the joint-probability distribution for degrees of adjacent vertices. For $s \in \mathcal{S}$, write $N_{k, l}^{(s, s)}(t)$ for the number of adjacent pairs of vertices with type $s$ whose degrees are $k$ and $l$, respectively, at time $t$ and $N_{k, l}^{(s, 1-s)}(t)$ for the number of vertices of degree $k$ with type $s$ which attach to a vertex of degree $l$ with type $1-s$ at time $t . N_{k}^{(s)}(t)$ is defined as before. For $\delta=0$, we have the following.

Theorem 9. (i) In our model, for $s \in \mathcal{S}$, the joint degree distributions of pairs of adjacent vertices with the same type are

$$
\begin{aligned}
P_{k, l}^{(s, s)}= & \lim _{t \rightarrow \infty} \frac{E\left[N_{k, l}^{(s, s)}(t)\right]}{t+1} \\
= & \Gamma\left(k+\frac{\beta\left(1-q_{s}\right)\left[(1+\alpha) q_{s}+\beta\left(1-q_{s}\right)\right]}{\alpha q_{s}^{2}}\right) \\
& \times \Gamma\left(l+\frac{\beta\left(1-q_{s}\right)\left[(1+\alpha) q_{s}+\beta\left(1-q_{s}\right)\right]}{\alpha q_{s}^{2}}\right) \\
& \times\left(\Gamma \left(k+l+1+\frac{(1+\alpha) q_{s}+\beta\left(1-q_{s}\right)}{\alpha q_{s}}\right.\right. \\
& \left.\left.+\frac{2 \beta\left(1-q_{s}\right)\left[(1+\alpha) q_{s}+\beta\left(1-q_{s}\right)\right]}{\alpha q_{s}^{2}}\right)\right)^{-1} \\
& \gamma^{(s, s)} \sum_{j=0}^{l-2} \frac{\Gamma(k+l-j-2)}{\Gamma(l-j-1) \Gamma(k)} \pi^{(s, s)}(j+2) ;
\end{aligned}
$$


for $k \geq 1, l \geq 2$, the vertex of type $s$ with degree $k$ is younger than the vertex of type $s$ with degreel, where

$$
\begin{aligned}
& \gamma^{(s, s)} \\
& =\Gamma\left(2+\frac{(1+\alpha) q_{s}+\beta\left(1-q_{s}\right)}{\alpha q_{s}}\right. \\
& \left.\quad+\frac{\beta\left(1-q_{s}\right)\left[(1+\alpha) q_{s}+\beta\left(1-q_{s}\right)\right]}{\alpha q_{s}^{2}}\right) \\
& \quad \times\left(\left[\Gamma\left(1+\frac{\beta\left(1-q_{s}\right)\left[(1+\alpha) q_{s}+\beta\left(1-q_{s}\right)\right]}{\alpha q_{s}^{2}}\right)\right]^{2}\right)^{-1} \\
& \quad \times P_{1}^{(s)},
\end{aligned}
$$

and for all $l \geq 2$

$$
\begin{aligned}
& \pi^{(s, s)}(l) \\
& =\Gamma\left(1+l+\frac{(1+\alpha) q_{s}+\beta\left(1-q_{s}\right)}{\alpha q_{s}}\right. \\
& \left.+\frac{2 \beta\left(1-q_{s}\right)\left[(1+\alpha) q_{s}+\beta\left(1-q_{s}\right)\right]}{\alpha q_{s}^{2}}\right) \\
& \times\left(\Gamma \left(l+\frac{(1+\alpha) q_{s}+\beta\left(1-q_{s}\right)}{\alpha q_{s}}\right.\right. \\
& \left.\left.+\frac{\beta\left(1-q_{s}\right)\left[(1+\alpha) q_{s}+\beta\left(1-q_{s}\right)\right]}{\alpha q_{s}^{2}}\right)\right)^{-1} \\
& \cdot(l-1) \\
& \times\left(l-1+\frac{\beta\left(1-q_{s}\right)\left[(1+\alpha) q_{s}+\beta\left(1-q_{s}\right)\right]}{\alpha q_{s}^{2}}\right)^{-1} .
\end{aligned}
$$

(ii) For $q_{0}=q_{1}=1 / 2$, one also has

$$
\begin{aligned}
P_{k, l}^{(s, 1-s)}= & \lim _{t \rightarrow \infty} \frac{E\left[N_{k, l}^{(s, 1-s)}(t)\right]}{t+1} \\
= & \frac{\Gamma(k+2 \beta / \alpha) \Gamma(l+2 \beta / \alpha)}{\Gamma(k+l+1+2 / \alpha+4 \beta / \alpha)} \\
& \cdot \gamma^{(s, 1-s)} \sum_{j=0}^{l-2} \frac{\Gamma(k+l-j-2)}{\Gamma(l-j-1) \Gamma(k)} \pi^{(s, 1-s)}(j+2),
\end{aligned}
$$

where

$$
\begin{gathered}
\gamma^{(s, 1-s)}=\frac{\Gamma(2+2(1+\beta) / \alpha)}{[\Gamma(1+2 \beta / \alpha)]^{2}} \cdot \frac{2 \beta}{\alpha[\alpha+2(1+\beta)]}, \\
\pi^{(s, 1-s)}(l)=\frac{1}{l-1+2 \beta / \alpha} \cdot \frac{\Gamma(l+1+2(1+2 \beta) / \alpha)}{\Gamma(l+2(1+2 \beta) / \alpha)},
\end{gathered}
$$

for $k \geq 1, l \geq 2$, when the vertex of type $s$ with degree $k$ is younger than the vertex of type $1-s$ with degreel.
The rest of this paper is organized as follows. In Section 2, we state four lemmas which are useful to prove our main results. Especially, in Lemma 12 we compute the expectation of the total degree of vertices with type $s$ and the total number of vertices with type $s$, respectively. In Lemma 13 we give the moment inequalities for the total degree of vertices with type $s$ and the total number of vertices with type $s$. In Section 3, we give the proofs of our main results in Theorems 3 and 8 . In Section 4, we prove our main result about joint-probability distribution for degrees of adjacent vertices with the same type and different types, respectively. In the appendix, we give the proof of Lemmas 12 and 13.

\section{Preliminaries}

The following two lemmas are useful to prove our main results. The readers who are interested in their details can refer to the associated materials.

Lemma 10 (see $[15])$. Suppose that a sequence $\left\{a_{t}\right\}$ satisfies the recurrence relation

$$
a_{t+1}=\left(1-\frac{b_{t}}{t+t_{1}}\right) a_{t}+c_{t}
$$

for $t \geq t_{0}$. Furthermore, suppose that $\lim _{t \rightarrow \infty} b_{t}=b>0$ and $\lim _{t \rightarrow \infty} c_{t}=c$. Then $\lim _{t \rightarrow \infty}\left(a_{t} / t\right)$ exists and

$$
\lim _{t \rightarrow \infty} \frac{a_{t}}{t}=\frac{c}{1+b} \text {. }
$$

Lemma 11 (see [11]). Let $p \in[1,2]$ and suppose that $\left\{X_{i} ; i \geq\right.$ $1\}$ are i.i.d. sequence with $E\left[X_{1}\right]=0$ and $E\left[\left|X_{1}\right|^{p}\right]<\infty$. Then there exists a constant $c_{p}$ depending only on $p$, such that

$$
E\left[\left|\sum_{i=1}^{t} X_{i}\right|^{p}\right] \leq c_{p} t E\left[\left|X_{1}\right|^{p}\right] .
$$

In the following, in graph $G(t-1)$, we denote the total degree of vertices with type $s$ by $S_{t-1}^{(s)}$, that is,

$$
S_{t-1}^{(s)}=\sum_{i=0}^{t-1} d_{i}^{(s)}(t-1),
$$

and the number of vertices of type $s$ by $T_{t-1}^{(s)}$; that is,

$$
T_{t-1}^{(s)}=\sum_{i=0}^{t-1} I_{\left\{Y_{i}=s\right\}}
$$

Now we conclude this section by stating the following two lemmas whose proofs are proposed in the appendix.

Lemma 12. For $s=0,1$, one has the following:

(i) $E\left[S_{t-1}^{(s)}\right]=1+q_{s}(t-2)+\left[\alpha q_{s}+\beta\left(1-q_{s}\right)\right](t-2)$.

(ii) $E\left[T_{t-1}^{(s)}\right]=1+q_{s}(t-2)$. 
Lemma 13. Let $S_{t-1}^{(s)}$ and $T_{t-1}^{(s)}$ be defined as before; then

(i) for $\forall 0<\varepsilon<1$, there exists a constant $C$ which is independent of the parameter $t$ such that

(ii) one has the following:

$$
\begin{gathered}
E\left|T_{t-1}^{(s)}-E\left[T_{t-1}^{(s)}\right]\right| \leq C t^{1 /(1+\varepsilon)}, \\
E\left|S_{t-1}^{(s)}-E\left[S_{t-1}^{(s)}\right]\right| \leq 2 \sqrt{t}
\end{gathered}
$$

\section{Proof of Theorems 3 and 8}

3.1. The Master Equations for the Degree Sequences of Vertices with Two Different Types. For $t$ large enough, it is easy to obtain two master equations of the degree sequences of different types as follows:

$$
\begin{aligned}
& E\left[N_{k}^{(s)}(t)-N_{k}^{(s)}(t-1) \mid \mathscr{F}_{t-1}\right] \\
& =-\left[\frac{\alpha(k+\delta) q_{s}}{S_{t-1}^{(s)}+\delta T_{t-1}^{(s)}}+\frac{\beta\left(1-q_{s}\right)}{T_{t-1}^{(s)}}\right] N_{k}^{(s)}(t-1) \\
& +\left[\frac{\alpha(k-1+\delta) q_{s}}{S_{t-1}^{(s)}+\delta T_{t-1}^{(s)}}+\frac{\beta\left(1-q_{s}\right)}{T_{t-1}^{(s)}}\right] N_{k-1}^{(s)}(t-1) \\
& +\delta_{k, 1} I_{\left\{Y_{t}=s\right\}} \quad s=0,1 .
\end{aligned}
$$

Now let us come to solve (27). Taking expectation of both sides of above equation and rewriting it, we get

$$
\begin{aligned}
& E\left[N_{k}^{(s)}(t)\right]\left\{1-\frac{\alpha(k+\delta) q_{s}}{E\left[S_{t-1}^{(s)}+\delta T_{t-1}^{(s)}\right]}-\frac{\beta\left(1-q_{s}\right)}{E\left[T_{t-1}^{(s)}\right]}\right\} E\left[N_{k}^{(s)}(t-1)\right] \\
&+\left\{\frac{\alpha(k-1+\delta) q_{s}}{E\left[S_{t-1}^{(s)}+\delta T_{t-1}^{(s)}\right]}+\frac{\beta\left(1-q_{s}\right)}{E\left[T_{t-1}^{(s)}\right]}\right\} E\left[N_{k-1}^{(s)}(t-1)\right] \\
&+\delta_{k, 1} q_{s}+\alpha q_{s}(k+\delta) \\
& \times E\left\{N_{k}^{(s)}(t-1) \frac{S_{t-1}^{(s)}+\delta T_{t-1}^{(s)}-E\left[S_{t-1}^{(s)}+\delta T_{t-1}^{(s)}\right]}{E\left[S_{t-1}^{(s)}+\delta T_{t-1}^{(s)}\right] \cdot\left(S_{t-1}^{(s)}+\delta T_{t-1}^{(s)}\right)}\right\} \\
&-\alpha q_{s}(k-1+\delta) \\
& \times E\left\{N_{k-1}^{(s)}(t-1) \frac{S_{t-1}^{(s)}+\delta T_{t-1}^{(s)}-E\left[S_{t-1}^{(s)}+\delta T_{t-1}^{(s)}\right]}{E\left[S_{t-1}^{(s)}+\delta T_{t-1}^{(s)}\right] \cdot\left(S_{t-1}^{(s)}+\delta T_{t-1}^{(s)}\right)}\right\} \\
&+\beta\left(1-q_{s}\right) E\left\{N_{k}^{(s)}(t-1) \frac{T_{t-1}^{(s)}-E\left[T_{t-1}^{(s)}\right]}{T_{t-1}^{(s)} \cdot E\left[T_{t-1}^{(s)}\right]}\right\} \\
& \beta\left\{\frac{T_{t-1}^{(s)}-E\left[T_{t-1}^{(s)}\right]}{T_{t-1}^{(s)} \cdot E\left[T_{t-1}^{(s)}\right]}\right\} \\
& N_{k-1}^{(s)}(t-1-
\end{aligned}
$$

Note that, for any $k \geq 1$ and $s=0,1$, we have the following inequalities:

$$
\begin{aligned}
& \left|\frac{N_{k}^{(s)}(t-1)}{S_{t-1}^{(s)}+\delta T_{t-1}^{(s)}}\right| \leq 1, \\
& \left|\frac{N_{k}^{(s)}(t-1)}{T_{t-1}^{(s)}}\right| \leq 1 .
\end{aligned}
$$

Combining Lemma 12, Lemma 13, triangle inequality, and (28)-(30), the last four sets of terms of (28) tend to zero as $t \rightarrow \infty$; then for $t$ large enough, we have

$$
\begin{aligned}
& E\left[N_{k}^{(s)}(t)\right] \\
& =\left[1-\frac{\alpha(k+\delta) q_{s}}{E\left[S_{t-1}^{(s)}+\delta T_{t-1}^{(s)}\right]}-\frac{\beta\left(1-q_{s}\right)}{E\left[T_{t-1}^{(s)}\right]}\right] E\left[N_{k}^{(s)}(t-1)\right] \\
& +\left[\frac{\alpha(k-1+\delta) q_{s}}{E\left[S_{t-1}^{(s)}+\delta T_{t-1}^{(s)}\right]}+\frac{\beta\left(1-q_{s}\right)}{E\left[T_{t-1}^{(s)}\right]}\right] E\left[N_{k-1}^{(s)}(t-1)\right] \\
& +\delta_{k, 1} q_{s}+o(1) \\
& =\left[1-\frac{\alpha(k+\delta) q_{s}}{1+\delta+\left[(1+\delta+\alpha) q_{s}+\beta\left(1-q_{s}\right)\right](t-2)}\right. \\
& \left.-\frac{\beta\left(1-q_{s}\right)}{1+q_{s}(t-2)}\right] E\left[N_{k}^{(s)}(t-1)\right]+\delta_{k, 1} q_{s} \\
& +\left[\frac{\alpha(k-1+\delta) q_{s}}{1+\delta+\left[(1+\delta+\alpha) q_{s}+\beta\left(1-q_{s}\right)\right](t-2)}\right. \\
& \left.+\frac{\beta\left(1-q_{s}\right)}{1+q_{s}(t-2)}\right] E\left[N_{k-1}^{(s)}(t-1)\right]+o(1) \text {. }
\end{aligned}
$$

For $k=1$, all $t>0$, note that we have $N_{0}^{(s)}(t)=0$ for $s \in \mathcal{S}$; thus combining (31) we obtain

$$
E\left[N_{1}^{(s)}(t)\right]=\left(1-\frac{b_{t}}{t}\right) E\left[N_{1}^{(s)}(t-1)\right]+q_{s}+o(1),
$$

where

$$
\begin{aligned}
b_{t}= & \frac{\alpha(1+\delta) q_{s}}{(1+\delta)(1 / t)+\left[(1+\delta+\alpha) q_{s}+\beta\left(1-q_{s}\right)\right]((t-2) / t)} \\
& +\frac{\beta\left(1-q_{s}\right)}{1 / t+q_{s}((t-2) / t)} .
\end{aligned}
$$


For $k>1$ and all $t>0$, according to (31), and for $s \in \mathcal{S}$, respectively, we also have

$$
\begin{aligned}
& E\left[N_{k}^{(s)}(t)\right] \\
& =\left[1-\frac{\alpha(k+\delta) q_{s}}{1+\delta+\left[(1+\delta+\alpha) q_{s}+\beta\left(1-q_{s}\right)\right](t-2)}\right. \\
& \left.\quad-\frac{\beta\left(1-q_{s}\right)}{1+q_{s}(t-2)}\right] E\left[N_{k}^{(s)}(t-1)\right] \\
& +\left[\frac{\alpha(k-1+\delta) q_{s}}{1+\delta+\left[(1+\delta+\alpha) q_{s}+\beta\left(1-q_{s}\right)\right](t-2)}\right. \\
& \left.\quad+\frac{\beta\left(1-q_{s}\right)}{1+q_{s}(t-2)}\right] E\left[N_{k-1}^{(s)}(t-1)\right]+o(1) .
\end{aligned}
$$

To solve the above two equations ((32) and (34)), we want to show that the expected values $E\left[N_{k}^{(s)}(t)\right] /(t+1)$ follow power laws as $t$ goes to infinity. To see it, we proceed by induction on $k$ to show that the limits $\lim _{t \rightarrow \infty}\left(E\left[N_{k}^{(s)}(t)\right] /(t+\right.$ 1)) exist for each $k \geq 1$.

\subsection{Proof of Theorem 3}

Proof of Theorem 3. For $k=1$, according to (33), we have

$$
\begin{array}{r}
b_{t} \longrightarrow \frac{\alpha(1+\delta) q_{s}}{(1+\delta+\alpha) q_{s}+\beta\left(1-q_{s}\right)}+\frac{\beta\left(1-q_{s}\right)}{q_{s}} \\
\text { as } t \longrightarrow \infty,
\end{array}
$$

Thus we apply Lemma 10 with $c_{t} \rightarrow q_{s}=c($ as $t \rightarrow \infty)$ to obtain that $\lim _{t \rightarrow \infty}\left(E\left[N_{1}^{(s)}(t)\right] /(t+1)\right)$ exists and

$$
\begin{gathered}
P_{1}^{(s)}=\lim _{t \rightarrow \infty} \frac{E\left[N_{1}^{(s)}(t)\right]}{t+1} \\
=q_{s} \times\left(1+\frac{\alpha(1+\delta) q_{s}}{(1+\delta+\alpha) q_{s}+\beta\left(1-q_{s}\right)}\right. \\
\left.+\frac{\beta\left(1-q_{s}\right)}{q_{s}}\right)^{-1} .
\end{gathered}
$$

We suppose that $\lim _{t \rightarrow \infty}\left(E\left[N_{k-1}^{(s)}(t)\right] /(t+1)\right)=P_{k-1}^{(s)}$ exists; we use Lemma 10 again with

$$
\begin{aligned}
b_{t}= & \frac{\alpha(k+\delta) q_{s}}{(1+\delta)(1 / t)+\left[(1+\delta+\alpha) q_{s}+\beta\left(1-q_{s}\right)\right]((t-2) / t)} \\
& +\frac{\beta\left(1-q_{s}\right)}{1 / t+q_{s}((t-2) / t)}
\end{aligned}
$$

$$
\begin{aligned}
& \longrightarrow \frac{\alpha(k+\delta) q_{s}}{(1+\delta+\alpha) q_{s}+\beta\left(1-q_{s}\right)}+\frac{\beta\left(1-q_{s}\right)}{q_{s}}=b \\
& \text { as } t \rightarrow \infty, \\
& c_{t}=\left[\frac{\alpha(k-1+\delta) q_{s}}{(1+\delta)(1 / t)+\left[(1+\delta+\alpha) q_{s}+\beta\left(1-q_{s}\right)\right]((t-2) / t)}\right. \\
&\left.+\frac{\beta\left(1-q_{s}\right)}{1 / t+q_{s}((t-2) / t)}\right] \frac{E\left[N_{k-1}^{(s)}(t-1)\right]}{t} \\
& \longrightarrow\left[\frac{\alpha(k-1+\delta) q_{s}}{(1+\delta+\alpha) q_{s}+\beta\left(1-q_{s}\right)}+\frac{\beta\left(1-q_{s}\right)}{q_{s}}\right] P_{k-1}^{(s)}=c
\end{aligned}
$$

Then we can arrive at the $\operatorname{limit}_{\lim _{t \rightarrow \infty}}\left(E\left[N_{k}^{(s)}(t)\right] /(t+1)\right)$ which exists and is equal to

$$
\begin{aligned}
& P_{k}^{(s)} \\
& =\left(k-1+\delta+\frac{\beta\left(1-q_{s}\right)\left[(1+\delta+\alpha) q_{s}+\beta\left(1-q_{s}\right)\right]}{\alpha q_{s}^{2}}\right. \\
& \quad \times\left(k+\delta+\frac{(1+\delta+\alpha) q_{s}+\beta\left(1-q_{s}\right)}{\alpha q_{s}}\right. \\
& \left.\left.\quad+\frac{\beta\left(1-q_{s}\right)\left[(1+\delta+\alpha) q_{s}+\beta\left(1-q_{s}\right)\right]}{\alpha q_{s}^{2}}\right)^{-1}\right) P_{k-1}^{(s)} .
\end{aligned}
$$

Thus we can get (5) immediately, and (6) is a direct result of (5).

By Stirling's formula, we have that $\Gamma(k+a) / \Gamma(k) \sim$ $k^{a}$ as $k \rightarrow \infty$, from which it follows that $P_{k}^{(s)} \sim$ $c_{s} k^{-\left(2+\left((1+\delta) q_{s}+\beta\left(1-q_{s}\right)\right) / \alpha q_{s}\right)}(s=0,1)$ for some constants $c_{s}>$ 0 . Thus the power law exponent is $2+\left((1+\delta) q_{s}+\beta(1-\right.$ $\left.\left.q_{s}\right)\right) / \alpha q_{s}$.

3.3. Proof of Theorem 8. In our model, Azuma's inequality no longer works because there is no uniform bound on the change in the number of $N_{k}^{(s)}(t)$ when we investigate the influence of the extra information contained in $\mathscr{F}_{n}$ compared to the information contained in $\mathscr{F}_{n-1}$; that is, we have to bound the difference $\left|E\left[N_{k}^{(s)}(t) \mid \mathscr{F}_{n}\right]-E\left[N_{k}^{(s)}(t) \mid \mathscr{F}_{n-1}\right]\right|$. It is very difficult to do it for our model, so we use our method instead of Azuma's inequality, in which there is no need to use such a uniform bound.

Proof of Theorem 8. At first we note a basic fact as follows:

$$
\left|N_{k}^{(s)}(t+1)-N_{k}^{(s)}(t)\right| \leq 2 .
$$


At first, we consider the case $k=1$ for $s=0,1$, respectively. Consider

$$
\begin{aligned}
& \Delta_{1}^{(s)}(t+1) \\
& =E\left[N_{1}^{(s)}(t+1)-E\left[N_{1}^{(s)}(t+1)\right]\right]^{2} \\
& =E\left\{\left[N_{1}^{(s)}(t+1)-N_{1}^{(s)}(t)\right]+\left[N_{1}^{(s)}(t)-E\left[N_{1}^{(s)}(t)\right]\right]\right. \\
& \left.-\left[E\left[N_{1}^{(s)}(t+1)\right]-E\left[N_{1}^{(s)}(t)\right]\right]\right\}^{2} \\
& =E\left[N_{1}^{(s)}(t+1)-N_{1}^{(s)}(t)\right]^{2}+E\left[N_{1}^{(s)}(t)-E\left[N_{1}^{(s)}(t)\right]\right]^{2} \\
& +\left[E\left[N_{1}^{(s)}(t+1)\right]-E\left[N_{1}^{(s)}(t)\right]\right]^{2} \\
& +2 E\left\{\left[N_{1}^{(s)}(t+1)-N_{1}^{(s)}(t)\right] \cdot\left[N_{1}^{(s)}(t)-E\left[N_{1}^{(s)}(t)\right]\right]\right\} \\
& -2\left\{E\left[N_{1}^{(s)}(t+1)-N_{1}^{(s)}(t)\right]\right\}^{2} \\
& \leq E\left[N_{1}^{(s)}(t+1)-N_{1}^{(s)}(t)\right]^{2}+\Delta_{1}^{(s)}(t) \\
& +2 E\left\{\left[N_{1}^{(s)}(t+1)-N_{1}^{(s)}(t)\right] \cdot\left[N_{1}^{(s)}(t)-E\left[N_{1}^{(s)}(t)\right]\right]\right\} \\
& \leq 4+\Delta_{1}^{(s)}(t) \\
& +2 E\left[\left(N_{1}^{(s)}(t)-E\left[N_{1}^{(s)}(t)\right]\right)\right. \\
& \left.\cdot E\left[N_{1}^{(s)}(t+1)-N_{1}^{(s)}(t) \mid \mathscr{F}_{t}\right]\right] \\
& =4+\Delta_{1}^{(s)}(t) \\
& -2 E\left[\left(N_{1}^{(s)}(t)-E\left[N_{1}^{(s)}(t)\right]\right)\right. \\
& \left.\cdot\left(\alpha q_{s}(1+\delta) \frac{N_{1}^{(s)}(t)}{S_{t}^{(s)}+\delta T_{t}^{(s)}}+\beta\left(1-q_{s}\right) \frac{N_{1}^{(s)}(t)}{T_{t}^{(s)}}\right)\right] \\
& =4+\Delta_{1}^{(s)}(t)\left(1-\frac{2 \alpha q_{s}(1+\delta)}{E\left[S_{t}^{(s)}+\delta T_{t}^{(s)}\right]}-\frac{2 \beta\left(1-q_{s}\right)}{E\left[T_{t}^{(s)}\right]}\right) \\
& -2 \alpha q_{s}(1+\delta) \\
& \times E\left[\left(N_{1}^{(s)}(t)-E\left[N_{1}^{(s)}(t)\right]\right)\right. \\
& \left.\cdot\left(\frac{N_{1}^{(s)}(t)}{S_{t}^{(s)}+\delta T_{t}^{(s)}}-\frac{N_{1}^{(s)}(t)-E\left[N_{1}^{(s)}(t)\right]}{E\left[S_{t}^{(s)}+\delta T_{t}^{(s)}\right]}\right)\right] \\
& -2 \beta\left(1-q_{s}\right) E\left[\left(N_{1}^{(s)}(t)-E\left[N_{1}^{(s)}(t)\right]\right)\right. \\
& \left.\cdot\left(\frac{N_{1}^{(s)}(t)}{T_{t}^{(s)}}-\frac{N_{1}^{(s)}(t)-E\left[N_{1}^{(s)}(t)\right]}{E\left[T_{t}^{(s)}\right]}\right)\right] \\
& =4+\Delta_{1}^{(s)}(t)\left(1-\frac{2 \alpha q_{s}(1+\delta)}{E\left[S_{t}^{(s)}+\delta T_{t}^{(s)}\right]}-\frac{2 \beta\left(1-q_{s}\right)}{E\left[T_{t}^{(s)}\right]}\right)+I_{1}+I_{2} \text {, }
\end{aligned}
$$

where

$$
\begin{aligned}
& I_{1}=-2 \alpha q_{s}(1+\delta) \\
& \times E {\left[\left(N_{1}^{(s)}(t)-E\left[N_{1}^{(s)}(t)\right]\right)\right.} \\
&\left.\cdot\left(\frac{N_{1}^{(s)}(t)}{S_{t}^{(s)}+\delta T_{t}^{(s)}}-\frac{N_{1}^{(s)}(t)}{E\left[S_{t}^{(s)}+\delta T_{t}^{(s)}\right]}\right)\right], \\
& I_{2}=-2 \beta\left(1-q_{s}\right) E\left[\left(N_{1}^{(s)}(t)-E\left[N_{1}^{(s)}(t)\right]\right)\right. \\
&\left.\cdot\left(\frac{N_{1}^{(s)}(t)}{T_{t}^{(s)}}-\frac{N_{1}^{(s)}(t)}{E\left[T_{t}^{(s)}\right]}\right)\right] .
\end{aligned}
$$

Now let us come to estimate (41) and (42), respectively. For (41), we note the basic fact that

$$
0<\frac{(1+\delta) N_{1}^{(s)}(t)}{S_{t}^{(s)}+\delta T_{t}^{(s)}} \leq 1
$$

By Hölder's inequality, letting $\varepsilon=1$ in (A.8) and combining with (A.16) we can easily obtain

$$
\begin{aligned}
I_{1} \leq & 2 \alpha q_{s} \sqrt{E\left(N_{1}^{(s)}(t)-E\left[N_{1}^{(s)}(t)\right]\right)^{2}} \\
& \cdot \sqrt{\frac{E\left(S_{t}^{(s)}+\delta T_{t}^{(s)}-E\left[S_{t}^{(s)}+\delta T_{t}^{(s)}\right]\right)^{2}}{\left(E\left[S_{t}^{(s)}+\delta T_{t}^{(s)}\right]\right)^{2}}} \\
& \leq 2 \alpha q_{s} \sqrt{E\left(N_{1}^{(s)}(t)-E\left[N_{1}^{(s)}(t)\right]\right)^{2}} \cdot \frac{C_{1}}{\sqrt{t}} \\
& \leq \alpha q_{s}\left[\frac{E\left(N_{1}^{(s)}(t)-E\left[N_{1}^{(s)}(t)\right]\right)^{2}}{A_{1} t}+C_{1}^{2} A_{1}\right] \\
& =\alpha q_{s} \frac{\Delta_{1}^{(s)}(t)}{A_{1} t}+\alpha q_{s} C_{1}^{2} A_{1},
\end{aligned}
$$

where the constant $C_{1}$ is independent of the parameter $t$, and we can take a constant $A_{1}$ such that

$$
0<\frac{2(1+\delta)}{\max \left\{1+\delta,(1+\delta+\alpha) q_{s}+\beta\left(1-q_{s}\right)\right\}}-\frac{1}{A_{1}}<1 .
$$

Similarly there also exist constants $C_{2}$ (independent of the parameter $t$ ) and $A_{2}$ such that

$$
I_{2} \leq \beta\left(1-q_{s}\right) \frac{\Delta_{1}^{(s)}(t)}{A_{2} t}+\beta\left(1-q_{s}\right) C_{2}^{2} A_{2}
$$


where $0<2-\left(1 / A_{2}\right)<1$. We denote

$$
\begin{aligned}
\varphi_{1}^{(s)}= & \varphi_{1}^{(s)}\left(\delta, \alpha, C_{1}, A_{1}, C_{2}, A_{2}\right) \\
= & \alpha q_{s}\left[\frac{2(1+\delta)}{\max \left\{1+\delta,(1+\delta+\alpha) q_{s}+\beta\left(1-q_{s}\right)\right\}}-\frac{1}{A_{1}}\right] \\
& +\beta\left(1-q_{s}\right)\left(2-\frac{1}{A_{2}}\right), \\
& \theta_{1}^{(s)}=4+\alpha q_{s} C_{1}^{2} A_{1}+\beta\left(1-q_{s}\right) C_{2}^{2} A_{2},
\end{aligned}
$$

noting the initial condition that $N_{1}^{(s)}(1)=1$ so that $\Delta_{1}^{(s)}(1)=$ 0 ; then for $t$ large enough we have

$$
\begin{aligned}
& \Delta_{1}^{(s)}(t+1) \\
& \quad \leq\left(1-\frac{\varphi_{1}^{(s)}}{t}\right) \Delta_{1}^{(s)}(t)+\theta_{1}^{(s)} \\
& \quad \leq \sum_{k=2}^{t+1} \prod_{j=k}^{t}\left(1-\frac{\varphi_{1}^{(s)}}{j}\right) \theta_{1}^{(s)}
\end{aligned}
$$

(we let the vacant product be equal to 1 )

$$
\begin{aligned}
& =\sum_{k=2}^{t+1} \exp \left(\sum_{j=k}^{t} \ln \left(1-\frac{\varphi_{1}^{(s)}}{j}\right)\right) \theta_{1}^{(s)} \\
& \approx \sum_{k=2}^{t+1}\left(\frac{k}{t}\right)^{\varphi_{1}^{(s)}} \cdot \theta_{1}^{(s)} \\
& \approx t \theta_{1}^{(s)} \int_{0}^{1} x^{\varphi_{1}^{(s)}} d x \\
& \leq \frac{\theta_{1}^{(s)}}{1+\varphi_{1}^{(s)}}(t+1) .
\end{aligned}
$$

Thus it follows that

$$
E\left[\frac{N_{1}^{(s)}(t)}{t}-\frac{E\left[N_{1}^{(s)}(t)\right]}{t}\right]^{2} \leq \frac{\theta_{1}^{(s)}}{1+\varphi_{1}^{(s)}} \frac{1}{t}
$$

then we arrive at

$$
\begin{aligned}
& \sum_{n=1}^{\infty} \mathbf{P}\left(\left|\frac{N_{1}^{(s)}\left(n^{2}\right)}{n^{2}}-\frac{E\left[N_{1}^{(s)}\left(n^{2}\right)\right]}{n^{2}}\right| \geq \varepsilon\right) \\
& \quad \leq \frac{1}{\varepsilon^{2}} \sum_{n=1}^{\infty} E\left[\frac{N_{1}^{(s)}\left(n^{2}\right)}{n^{2}}-\frac{E\left[N_{1}^{(s)}\left(n^{2}\right)\right]}{n^{2}}\right]^{2} \\
& \quad \leq \frac{1}{\varepsilon^{2}} \frac{\theta_{1}^{(s)}}{1+\varphi_{1}^{(s)}} \sum_{n=1}^{\infty} \frac{1}{n^{2}}<\infty .
\end{aligned}
$$

Thus Theorem 3 and the Borel-Cantelli lemma imply that

$$
\frac{N_{1}^{(s)}\left(n^{2}\right)}{n^{2}} \longrightarrow P_{1}^{(s)} \quad \text { a.s. }
$$

where $P_{1}^{(s)}$ is defined by (6). For all $t$, we take $n^{2} \leq t \leq(n+1)^{2}$; we have

$$
\left|N_{1}^{(s)}(t)-N_{1}^{(s)}\left(n^{2}\right)\right| \leq 2\left(t-n^{2}\right) \leq 4 n+2 .
$$

Then we have

$$
\begin{aligned}
& \left|\frac{N_{1}^{(s)}(t)}{t}-\frac{N_{1}^{(s)}\left(n^{2}\right)}{n^{2}}\right| \\
& \leq\left|\frac{N_{1}^{(s)}(t)-N_{1}^{(s)}\left(n^{2}\right)}{t}\right|+\left|\frac{N_{1}^{(s)}\left(n^{2}\right)\left(t-n^{2}\right)}{t \cdot n^{2}}\right| \\
& \quad(\text { as } n \longrightarrow 0
\end{aligned}
$$

thus we get

$$
\frac{N_{1}^{(s)}(t)}{t} \longrightarrow P_{1}^{(s)} \quad \text { a.s. }
$$

Similarly to the case $k>1$ and $s=0,1$, we can also prove that

$$
\frac{N_{k}^{(s)}(t)}{t} \longrightarrow P_{k}^{(s)} \quad \text { a.s., }
$$

where $P_{k}^{(s)}$ is defined as in Theorem 3 .

\section{Proof of Theorem 9}

4.1. Master Equations for Joint Degree Sequences $N_{k, l}^{(s, s)}(t)$ and $N_{k, l}^{(s, 1-s)}(t)$. Let $\delta=0$; we can easily get the master equations as follows: for $s \in \mathcal{S}$

$$
\begin{aligned}
& E\left[N_{k, l}^{(s, s)}(t)-N_{k, l}^{(s, s)}(t-1) \mid \mathscr{F}_{t-1}\right] \\
& =\left[\frac{\alpha q_{s}(k-1)}{S_{t-1}^{(s)}}+\frac{\beta\left(1-q_{s}\right)}{T_{t-1}^{(s)}}\right] N_{k-1, l}^{(s, s)}(t-1) \\
& +\left[\frac{\alpha q_{s}(l-1)}{S_{t-1}^{(s)}}+\frac{\beta\left(1-q_{s}\right)}{T_{t-1}^{(s)}}\right] N_{k, l-1}^{(s, s)}(t-1) \\
& +\left[\frac{\alpha q_{s}(k+l)}{S_{t-1}^{(s)}}+\frac{2 \beta\left(1-q_{s}\right)}{T_{t-1}^{(s)}}\right] N_{k, l}^{(s, s)}(t-1) \\
& +\frac{\alpha q_{s}(l-1)}{S_{t-1}^{(s)}} N_{l-1}^{(s)}(t-1) \delta_{k, 1},
\end{aligned}
$$

where we suppose that the vertex of type $s$ with degree $k$ is younger than the vertex of type $s$ with degree $l$. The first two sets of terms on the right-hand side account for the change in $N_{k, l}^{(s, s)}(t)$ due to the addition of the new edge hitting a vertex of degree $k-1$ or $l-1$ (both gain) with type $s$, while the third set of terms gives the change in $N_{k, l}^{(s, s)}(t-1)$ due to the addition of the new edge onto the ancestor vertex of degree $k$ or $l$ (both loss) with type $s$. Finally, the last term accounts for the gain in $N_{1, l}^{(s, s)}(t-1)$ due to the addition on the new vertex. 
Similarly, for $s \in \mathcal{S}$ we also have

$$
\begin{aligned}
E[ & \left.N_{k, l}^{(s, 1-s)}(t)-N_{k, l}^{(s, 1-s)}(t-1) \mid \mathscr{F}_{t}\right] \\
= & {\left[\frac{\alpha q_{s}(k-1)}{S_{t-1}^{(s)}}+\frac{\beta\left(1-q_{s}\right)}{T_{t-1}^{(s)}}\right] N_{k-1, l}^{(s, 1-s)}(t-1) } \\
& +\left[\frac{\alpha\left(1-q_{s}\right)(l-1)}{S_{t-1}^{(1-s)}}+\frac{\beta q_{s}}{T_{t-1}^{(1-s)}}\right] N_{k, l-1}^{(s, 1-s)}(t-1) \\
& -\left[\frac{\alpha q_{s} k}{\left.S_{t-1}^{(s)}+\frac{\beta\left(1-q_{s}\right)}{T_{t-1}^{(s)}}\right] N_{k, l}^{(s, 1-s)}(t-1)}\right. \\
& -\left[\frac{\alpha\left(1-q_{s}\right) l}{S_{t-1}^{(1-s)}}+\frac{\beta q_{s}}{T_{t-1}^{(1-s)}}\right] N_{k, l}^{(s, 1-s)}(t-1) \\
& +\frac{\beta q_{s}}{T_{t-1}^{(1-s)}} N_{l-1}^{(1-s)}(t-1) \delta_{k, 1},
\end{aligned}
$$

where the vertex of type $s$ with degree $k$ is younger than the vertex of type $1-s$ with degree $l$.

We also notice a fact that the total number of pairs of adjacent vertices is equal to the number of edges at time $t$. The total number of edges is $t$ in the resulting graph $G(t)$; obviously, there exist constants $\theta_{i}(i=1,2,3)$ such that

$$
\begin{aligned}
& \left|\frac{N_{k, l}^{(s, s)}(t-1)}{S_{t-1}^{(s)}}\right| \leq 1, \quad\left|\frac{N_{k, l}^{(s, 1-s)}(t-1)}{S_{t-1}^{(s)}}\right| \leq 1, \\
& \left|\frac{N_{k, l}^{(s, 1-s)}(t)}{S_{t-1}^{(1-s)}}\right| \leq 1 . \\
& \left|\frac{N_{k, l}^{(s, s)}(t-1)}{T_{t-1}^{(s)}}\right| \leq \theta_{1}, \quad\left|\frac{N_{k, l}^{(s, 1-s)}(t)}{T_{t-1}^{(s)}}\right| \leq \theta_{2}, \\
& \left|\frac{N_{k, l}^{(s, 1-s)}(t)}{T_{t-1}^{(1-s)}}\right| \leq \theta_{3} .
\end{aligned}
$$

Similarly to the analysis of (27), for $t$ large enough, combining Lemmas 12 and 13 and (58), we can get by (56) and (57), respectively,

$$
\begin{aligned}
E & {\left[N_{k, l}^{(s, s)}(t)-N_{k, l}^{(s, s)}(t-1)\right] } \\
= & {\left[\frac{\alpha q_{s}(k-1)}{1+\left[(1+\alpha) q_{s}+\beta\left(1-q_{s}\right)\right](t-2)}+\frac{\beta\left(1-q_{s}\right)}{1+q_{s}(t-2)}\right] } \\
& \times E\left[N_{k-1, l}^{(s, s)}(t-1)\right] \\
& +\left[\frac{\alpha q_{s}(l-1)}{1+\left[(1+\alpha) q_{s}+\beta\left(1-q_{s}\right)\right](t-2)}+\frac{\beta\left(1-q_{s}\right)}{1+q_{s}(t-2)}\right] \\
& \times E\left[N_{k, l-1}^{(s, s)}(t-1)\right]
\end{aligned}
$$

$$
\begin{aligned}
& -\left[\frac{\alpha q_{s}(k+l)}{1+\left[(1+\alpha) q_{s}+\beta\left(1-q_{s}\right)\right](t-2)}+\frac{2 \beta\left(1-q_{s}\right)}{1+q_{s}(t-2)}\right] \\
& \times E\left[N_{k, l}^{(s, s)}(t-1)\right] \\
& +\frac{\alpha q_{s}(l-1)}{1+\left[(1+\alpha) q_{s}+\beta\left(1-q_{s}\right)\right](t-2)} E\left[N_{l-1}^{(s)}(t-1)\right] \\
& \times \delta_{k, 1}+O\left(t^{-1 / 2}\right) \text {, } \\
& E\left[N_{k, l}^{(s, 1-s)}(t)-N_{k, l}^{(s, 1-s)}(t-1)\right] \\
& =\left[\frac{\alpha q_{s}(k-1)}{1+\left[(1+\alpha) q_{s}+\beta\left(1-q_{s}\right)\right](t-2)}+\frac{\beta\left(1-q_{s}\right)}{1+q_{s}(t-2)}\right] \\
& \times E\left[N_{k-1, l}^{(s, 1-s)}(t-1)\right] \\
& +\left[\frac{\alpha\left(1-q_{s}\right)(l-1)}{1+\left[(1+\alpha)\left(1-q_{s}\right)+\beta q_{s}\right](t-2)}\right. \\
& \left.+\frac{\beta q_{s}}{1+\left(1-q_{s}\right)(t-2)}\right] E\left[N_{k, l-1}^{(s, 1-s)}(t-1)\right] \\
& -\left[\frac{\alpha q_{s} k}{1+\left[(1+\alpha) q_{s}+\beta\left(1-q_{s}\right)\right](t-2)}+\frac{\beta\left(1-q_{s}\right)}{1+q_{s}(t-2)}\right] \\
& \times E\left[N_{k, l}^{(s, 1-s)}(t-1)\right] \\
& -\left[\frac{\alpha\left(1-q_{s}\right) l}{1+\left[(1+\alpha)\left(1-q_{s}\right)+\beta q_{s}\right](t-2)}\right. \\
& \left.+\frac{\beta q_{s}}{1+\left(1-q_{s}\right)(t-2)}\right] E\left[N_{k, l}^{(s, 1-s)}(t-1)\right] \\
& +\frac{\beta q_{s}}{1+\left(1-q_{s}\right)(t-2)} E\left[N_{l-1}^{(1-s)}(t-1)\right] \delta_{k, 1}+O\left(t^{-1 / 2}\right) \text {. }
\end{aligned}
$$

4.2. Proof of Theorem 9. To solve the above equations ((59), (60), resp.), we come to prove Theorem 9 as follows.

Proof of Theorem 9. At first we have

$$
\begin{gathered}
N_{k, 1}^{(s, s)}(t)=0 \quad(\text { for } k \geq 1), \\
N_{0, l}^{(s, s)}(t)=0 \quad(\text { for } l \geq 2), \quad \text { for all } t \geq 1
\end{gathered}
$$

so that

$$
P_{k, 1}^{(s, s)}=0 \quad(\text { for } k \geq 1), \quad P_{0, l}^{(s, s)}=0 \quad(\text { for } l \geq 2) .
$$

Thus we have

$$
\begin{aligned}
E\left[N_{1,2}^{(s, s)}(t)\right] & \\
= & {\left[1-\frac{3 \alpha q_{s}}{1+\left[(1+\alpha) q_{s}+\beta\left(1-q_{s}\right)\right](t-2)}\right.} \\
& \left.\quad-\frac{2 \beta\left(1-q_{s}\right)}{1+q_{s}(t-2)}\right] E\left[N_{1,2}^{(s, s)}(t-1)\right] \\
& +\frac{\alpha q_{s}}{1+\left[(1+\alpha) q_{s}+\beta\left(1-q_{s}\right)\right](t-2)} E\left[N_{1}^{(s)}(t-1)\right] ;
\end{aligned}
$$


combining Lemma 10 with Theorem 3, it follows that

$$
\begin{aligned}
& P_{1,2}^{(s, s)} \\
& =\frac{\alpha q_{s} /\left((1+\alpha) q_{s}+\beta\left(1-q_{s}\right)\right)}{1+3 \alpha q_{s} /\left((1+\alpha) q_{s}+\beta\left(1-q_{s}\right)\right)+2 \beta\left(1-q_{s}\right) / q_{s}} \\
& \quad \times P_{1}^{(s)}
\end{aligned}
$$

here and thereafter $P_{1}^{(s)}$ is defined as (5) in Theorem 3 when $\delta=0$.

For $k \geq 2, l \geq 2$, by induction hypothesis, we suppose that

$$
\begin{gathered}
\lim _{t \rightarrow \infty} E \frac{\left[N_{k-1, l}^{(s, s)}(t)\right]}{t}=P_{k-1, l}^{(s, s)}, \\
\lim _{t \rightarrow \infty} E \frac{\left[N_{k, l-1}^{(s)}(t)\right]}{t}=P_{k, l-1}^{(s, s)} \quad \text { exist. }
\end{gathered}
$$

Thus by using Lemma 10 and Theorem 3 again, we arrive at $\lim _{t \rightarrow \infty}\left(E\left[N_{k, l}^{(s, s)}(t)\right] / t\right)=P_{k, l}^{(s, s)}$ existence and satisfy the following time-independent recursion relation:

$$
\begin{aligned}
& {\left[1+\frac{\alpha q_{s}(k+l)}{(1+\alpha) q_{s}+\beta\left(1-q_{s}\right)}+\frac{2 \beta\left(1-q_{s}\right)}{q_{s}}\right] P_{k, l}^{(s, s)}} \\
& =\left[\frac{\alpha q_{s}(k-1)}{(1+\alpha) q_{s}+\beta\left(1-q_{s}\right)}+\frac{\beta\left(1-q_{s}\right)}{q_{s}}\right] P_{k-1, l}^{(s, s)} \\
& \quad+\left[\frac{\alpha q_{s}(l-1)}{(1+\alpha) q_{s}+\beta\left(1-q_{s}\right)}+\frac{\beta\left(1-q_{s}\right)}{q_{s}}\right] P_{k, l-1}^{(s, s)} \\
& \quad+\frac{\alpha q_{s}(l-1)}{(1+\alpha) q_{s}+\beta\left(1-q_{s}\right)} P_{l-1}^{(s)} \delta_{k, 1},
\end{aligned}
$$

which is equivalent to

$$
\begin{aligned}
& {\left[k+l+\frac{(1+\alpha) q_{s}+\beta\left(1-q_{s}\right)}{\alpha q_{s}}\right.} \\
& \left.+\frac{2 \beta\left(1-q_{s}\right)\left[(1+\alpha) q_{s}+\beta\left(1-q_{s}\right)\right]}{\alpha q_{s}^{2}}\right] P_{k, l}^{(s, s)} \\
& =\left[k-1+\frac{\beta\left(1-q_{s}\right)\left[(1+\alpha) q_{s}+\beta\left(1-q_{s}\right)\right]}{\alpha q_{s}^{2}}\right] P_{k-1, l}^{(s, s)} \\
& \quad+\left[l-1+\frac{\beta\left(1-q_{s}\right)\left[(1+\alpha) q_{s}+\beta\left(1-q_{s}\right)\right]}{\alpha q_{s}^{2}}\right] P_{k, l-1}^{(s, s)} \\
& \quad+(l-1) P_{l-1}^{(s)} \delta_{k, 1} .
\end{aligned}
$$

Now let us come to solve the difference equation (67). Letting

$$
\begin{aligned}
& P_{k, l}^{(s, s)} \\
& =\Gamma\left(k+\frac{\beta\left(1-q_{s}\right)\left[(1+\alpha) q_{s}+\beta\left(1-q_{s}\right)\right]}{\alpha q_{s}^{2}}\right) \\
& \quad \times \Gamma\left(l+\frac{\beta\left(1-q_{s}\right)\left[(1+\alpha) q_{s}+\beta\left(1-q_{s}\right)\right]}{\alpha q_{s}^{2}}\right) \\
& \quad \times\left(\Gamma \left(k+l+1+\frac{(1+\alpha) q_{s}+\beta\left(1-q_{s}\right)}{\alpha q_{s}}\right.\right. \\
& \left.\left.\quad+\frac{2 \beta\left(1-q_{s}\right)\left[(1+\alpha) q_{s}+\beta\left(1-q_{s}\right)\right]}{\alpha q_{s}^{2}}\right)\right)^{-1} \\
& \quad \times M_{k, l}^{(s, s)},
\end{aligned}
$$

substituting the above equation (68) in (67), and combining (6), we arrive at

$$
\begin{aligned}
& M_{k, l}^{(s, s)} \\
& =M_{k-1, l}^{(s, s)}+M_{k, l-1}^{(s, s)} \\
& +\Gamma\left(1+l+\frac{(1+\alpha) q_{s}+\beta\left(1-q_{s}\right)}{\alpha q_{s}}\right. \\
& \left.\quad+\frac{2 \beta\left(1-\mathrm{q}_{s}\right)\left[(1+\alpha) q_{s}+\beta\left(1-q_{s}\right)\right]}{\alpha q_{s}^{2}}\right) \\
& \quad \times\left(\quad\left(1+\frac{\beta\left(1-q_{s}\right)\left[(1+\alpha) q_{s}+\beta\left(1-q_{s}\right)\right]}{\alpha q_{s}^{2}}\right)\right. \\
& \left.\quad \times \Gamma\left(l+\frac{\beta\left(1-q_{s}\right)\left[(1+\alpha) q_{s}+\beta\left(1-q_{s}\right)\right]}{\alpha q_{s}^{2}}\right)\right)^{-1} \\
& \quad \times(l-1) P_{l-1}^{(s)} \delta_{k, 1} \\
& =M_{k-1, l}^{(s, s)}+M_{k, l-1}^{(s, s)}+\gamma^{(s, s)} \pi^{(s, s)}(l) \delta_{k, 1},
\end{aligned}
$$

where $\gamma^{(s, s)}, \pi^{(s, s)}(l)$ are defined as (16) and (17) for all $l \geq 2$, respectively.

Moreover, by (69) we have

$$
M_{k, 1}^{(s, s)}=0 \quad(\text { for } k \geq 1), \quad M_{0, l}^{(s, s)}=0 \quad(\text { for } l \geq 2) .
$$

Now we define 2-dimensional generating function as follows:

$$
G^{(s, s)}(x, y)=\sum_{k=1}^{\infty} \sum_{l=2}^{\infty} M_{k, l}^{(s, s)} x^{k} y^{l} \quad|x|<1,|y|<1
$$


it follows that

$$
\begin{aligned}
G^{(s, s)}(x, y)= & \sum_{k-1=1}^{\infty} \sum_{l=2}^{\infty} M_{k-1, l}^{(s, s)} x^{k} y^{l} \\
& +\sum_{k=1}^{\infty} \sum_{l-1=2}^{\infty} M_{k, l-1}^{(s, s)} x^{k} y^{l}+\sum_{l=2}^{\infty} \gamma^{(s, s)} \pi^{(s, s)}(l) x y^{l} \\
= & x G^{(s, s)}(x, y)+y G^{(s, s)}(x, y) \\
& +\gamma^{(s, s)} x \sum_{l=2}^{\infty} \pi^{(s, s)}(l) y^{l}
\end{aligned}
$$

so that

$$
G^{(s, s)}(x, y)=\frac{\gamma^{(s, s)} x}{1-x-y} \sum_{l=2}^{\infty} \pi^{(s, s)}(l) y^{l} ;
$$

furthermore, taking $0<r<1$, for $|x+y| \leq r$, and expanding $G^{(s, s)}(x, y)$, then

$$
\begin{aligned}
& G^{(s, s)}(x, y) \\
& =\gamma^{(s, s)} x \sum_{n=0}^{\infty}(x+y)^{n} \sum_{l=2}^{\infty} \pi^{(s, s)}(l) y^{l} \\
& =\gamma^{(s, s)} x \sum_{n=0}^{\infty} \sum_{i=0}^{n} C_{n}^{i} x^{n-i} y^{i} \sum_{j=0}^{\infty} \pi^{(s, s)}(j+2) y^{j+2} \\
& =\gamma^{(s, s)} \sum_{k=1}^{\infty} \sum_{u=0}^{\infty} \sum_{i+j=u} C_{k+i-1}^{i} \pi^{(s, s)}(j+2) x^{k} y^{u+2} \\
& =\gamma^{(s, s)} \sum_{k=1}^{\infty} \sum_{l=2}^{\infty} \sum_{j=0}^{l-2} C_{k+l-j-3}^{l-j-2} \pi^{(s, s)}(j+2) x^{k} y^{l} \\
& =\gamma^{(s, s)} \sum_{k=1}^{\infty} \sum_{l=2}^{\infty} \sum_{j=0}^{l-2} \frac{\Gamma(k+l-j-2)}{\Gamma(l-j-1) \Gamma(k)} \pi^{(s, s)}(j+2) x^{k} y^{l} .
\end{aligned}
$$

Comparing (71) with (74), it follows that

$$
M_{k, l}^{(s, s)}=\gamma^{(s, s)} \sum_{j=0}^{l-2} \frac{\Gamma(k+l-j-2)}{\Gamma(l-j-1) \Gamma(k)} \pi^{(s, s)}(j+2),
$$

so we arrive at (10) immediately. Furthermore, if $q_{0}=$ $q_{1}=1 / 2$, similarly to the analysis of (59), by doing the same procedure to (60), (13) can be easily derived. Thus we complete the proof of Theorem 9 .

\section{Appendix}

Proof of Lemma 12. For $i \geq 0, k \geq i+1$, and $s=0,1$, we define a sequence of random variables $\left\{\xi_{i, k}^{(s)}\right\}$ as follows:

$$
\xi_{i, k}^{(s)}= \begin{cases}1, & \text { if the new edge associated with the } k \text { th } \\ & \text { vertex hit the } i \text { th vertex of type } s\end{cases}
$$

It is simple to see that

$$
\begin{aligned}
& d_{0}^{(0)}(t-1)=1+\sum_{k=2}^{t-1} \xi_{0, k}^{(0)}, \\
& d_{1}^{(1)}(t-1)=1+\sum_{k=2}^{t-1} \xi_{1, k}^{(1)} .
\end{aligned}
$$

For $i \geq 2$,

$$
d_{i}^{(s)}(t-1)=I_{\left\{Y_{i}=s\right\}}+\sum_{k=i+1}^{t-1} \xi_{i, k}^{(s)}
$$

Moreover combining (3) and (4), we have

$$
\begin{aligned}
& P\left(\xi_{i, k}^{(s)}=1 \mid \mathscr{F}_{k-1}\right) \\
&= P\left(v_{k} \longrightarrow v_{i}, Y_{i}=s, Y_{k}=s \mid \mathscr{F}_{k-1}\right) \\
&+P\left(v_{k} \longrightarrow v_{i}, Y_{i}=s, Y_{k}=1-s \mid \mathscr{F}_{k-1}\right) \\
&= \alpha q_{s} \frac{d_{i}^{(s)}(k-1)+\delta^{(i, s)}}{S_{k-1}^{(s)}+\delta T_{k-1}^{(s)}}+\beta\left(1-q_{s}\right) \frac{I_{\left\{Y_{i}=s\right\}}}{T_{k-1}^{(s)}},
\end{aligned}
$$

so for the case $s=0$,

$$
\begin{aligned}
E & {\left[\sum_{i=0}^{t-1} d_{i}^{(0)}(t-1)\right] } \\
= & 1+E\left[\sum_{k=2}^{t-1} \xi_{0, k}^{(0)}\right]+E\left[\sum_{i=2}^{t-1} I_{\left\{Y_{i}=0\right\}}\right]+E\left[\sum_{i=2}^{t-1} \sum_{k=i+1}^{t-1} \xi_{i, k}^{(0)}\right] \\
= & 1+q_{0}(t-2)+E\left[\sum_{k=2}^{t-1} P\left(\xi_{0, k}^{(0)}=1 \mid \mathscr{F}_{k-1}\right)\right] \\
& +E\left[\sum_{i=2}^{t-1} \sum_{k=i+1}^{t-1} P\left(\xi_{i, k}^{(0)}=1 \mid \mathscr{F}_{k-1}\right)\right] \\
= & +q_{0}(t-2) \\
& +E \sum_{k=2}^{t-1}\left[\alpha q_{0} \frac{d_{0}^{(0)}(k-1)+\delta^{(0,0)}}{S_{k-1}^{(0)}+\delta T_{k-1}^{(0)}}+\beta\left(1-q_{0}\right) \frac{I_{\left\{Y_{0}=0\right\}}}{T_{k-1}^{(0)}}\right] \\
& +E \sum_{i=2}^{t-1} \sum_{k=i+1}^{t-1}\left[\alpha q_{0} \frac{d_{i}^{(0)}(k-1)+\delta^{(i, 0)}}{S_{k-1}^{(0)}+\delta T_{k-1}^{(0)}}+\beta\left(1-q_{0}\right) \frac{I_{\left\{Y_{i}=0\right\}}}{T_{k-1}^{(0)}}\right] \\
= & 1+q_{0}(t-2) \\
& +E \sum_{k=2}^{t-1}\left[\alpha q_{0} \frac{d_{0}^{(0)}(k-1)+\delta^{(0,0)}}{S_{k-1}^{(0)}+\delta T_{k-1}^{(0)}}+\beta\left(1-q_{0}\right) \frac{I_{\left\{Y_{0}=0\right\}}}{T_{k-1}^{(0)}}\right]
\end{aligned}
$$




$$
\begin{aligned}
&+E {\left[\sum_{k=3}^{t-1} \sum_{i=2}^{k-1} \alpha q_{0} \frac{d_{i}^{(0)}(k-1)+\delta^{(i, 0)}}{S_{k-1}^{(0)}+\delta T_{k-1}^{(0)}}\right.} \\
&\left.+\sum_{k=3}^{t-1} \sum_{i=2}^{k-1} \beta\left(1-q_{0}\right) \frac{I_{\left\{Y_{i}=0\right\}}}{T_{k-1}^{(0)}}\right] \\
&=1+q_{0}(t-2)+\left[\alpha q_{0}+\beta\left(1-q_{0}\right)\right](t-2) .
\end{aligned}
$$

Similarly we can get

$$
\begin{aligned}
& E\left[\sum_{i=0}^{t-1} d_{i}^{(1)}(t-1)\right] \\
& \quad=1+q_{1}(t-2)+\left[\alpha q_{1}+\beta\left(1-q_{1}\right)\right](t-2) .
\end{aligned}
$$

Thus (i) holds. Assertion (ii) is obvious since the $\left\{Y_{i} ; i \geq 2\right\}$ are i.i.d. sequence.

Proof of Lemma 13. (i) Applying Lemma 11 to $X_{i}=I_{\left\{Y_{i}=s\right\}}-q_{s}$ and combining with Hölder's inequality we have for $\forall \varepsilon>0$ and $p=1+\varepsilon$

$$
\begin{aligned}
& E\left|T_{t-1}^{(s)}-E\left[T_{t-1}^{(s)}\right]\right| \\
& \leq\left[E\left|\sum_{i=2}^{t-1}\left(I_{\left\{Y_{i}=s\right\}}-q_{s}\right)\right|^{1+\varepsilon}\right]^{1 /(1+\varepsilon)} \\
& \quad \leq\left\{c_{1+\varepsilon} t E\left[\left|I_{\left\{Y_{2}=s\right\}}-q_{s}\right|^{1+\varepsilon}\right]\right\}^{1 /(1+\varepsilon)} \\
& \quad \leq C t^{1 /(1+\varepsilon)}
\end{aligned}
$$

where $C=\left\{c_{1+\varepsilon}\left[\left(1-q_{s}\right)^{1+\varepsilon} q_{s}+q_{s}^{1+\varepsilon}\left(1-q_{s}\right)\right]\right\}^{1 /(1+\varepsilon)}$.

(ii) We introduce for $s=0,1$

$$
Z_{n}^{(s)}=E\left[S_{t-1}^{(s)} \mid \mathscr{F}_{n}\right], \quad n=0,1, \ldots, t-1,
$$

where $\mathscr{F}_{0}$ is $\sigma$-field generated by the empty graph. By Lemma 12 we have $E\left[Z_{n}^{(s)}\right]<\infty$; the process is a Doob martingale with respect to $\left\{\mathscr{F}_{n}\right\}_{n=0}^{t-1}$. Moreover, we have $Z_{t-1}^{(s)}=$ $S_{t-1}^{(s)}$ and $Z_{0}^{(s)}=E\left[S_{t-1}^{(s)}\right]$, so that

$$
S_{t-1}^{(s)}-E\left[S_{t-1}^{(s)}\right]=Z_{t-1}^{(s)}-Z_{0}^{(s)}
$$

Furthermore, we claim that

$$
\left|Z_{n}^{(s)}-Z_{n-1}^{(s)}\right| \leq 2
$$

To see this, we at first consider the case $s=0$, noticing that

$$
S_{t-1}^{(0)}=1+\sum_{i=2}^{t-1} I_{\left\{Y_{i}=0\right\}}+\sum_{k=2}^{t-1} \xi_{0, k}^{(0)}+\sum_{i=2}^{t-1} \sum_{k=i+1}^{t-1} \xi_{i, k}^{(0)}
$$

so that

$$
\begin{aligned}
Z_{n}^{(0)}= & E\left[S_{t-1}^{(0)} \mid \mathscr{F}_{n}\right] \\
= & E\left[1+\sum_{i=2}^{t-1} I_{\left\{Y_{i}=0\right\}}+\sum_{k=2}^{t-1} \xi_{0, k}^{(0)}+\sum_{i=2}^{t-1} \sum_{k=i+1}^{t-1} \xi_{i, k}^{(0)} \mid \mathscr{F}_{n}\right] \\
= & 1+\sum_{i=2}^{n} I_{\left\{Y_{i}=0\right\}}+q_{0}(t-n-1) \\
& +E\left[\sum_{k=2}^{t-1} \xi_{0, k}^{(0)} \mid \mathscr{F}_{n}\right]+E\left[\sum_{i=2}^{t-1} \sum_{k=i+1}^{t-1} \xi_{i, k}^{(0)} \mid \mathscr{F}_{n}\right] \\
= & +\sum_{i=2}^{n} I_{\left\{Y_{i}=0\right\}}+q_{0}(t-n-1) \\
& +\sum_{k=2}^{n} \xi_{0, k}^{(0)}+\sum_{k=n+1}^{t-1} E\left[E\left[\xi_{0, k}^{(0)} \mid \mathscr{F}_{k-1}\right] \mid \mathscr{F}_{n}\right] \\
& +\sum_{k=3}^{n} \sum_{i=2}^{n} \sum_{k=3}^{k-1} \xi_{i, k}^{(0)} \xi_{i, k}^{(0)}+\left[\alpha q_{0}+\beta\left(1-q_{0}\right)\right](t-n-1) \\
& +\sum_{k=n+1}^{t-1} \sum_{i=2}^{k-1} E\left[E\left[\xi_{i, k}^{(0)} \mid \mathscr{F}_{k-1}\right] \mid \mathscr{F}_{n}\right] \\
= & +\sum_{i=2}^{n} I_{\left\{Y_{i}=0\right\}}+q_{0}(t-n-1)+\sum_{k=2}^{n} \xi_{0, k}^{(0)}
\end{aligned}
$$

It follows that

$$
\begin{aligned}
& \left|Z_{n}^{(0)}-Z_{n-1}^{(0)}\right| \\
& \quad=\left|I_{\left\{Y_{n}=0\right\}}-q_{0}+\xi_{0, n}^{(0)}+\sum_{i=2}^{n-1} \xi_{i, n}^{(0)}-\left[\alpha q_{0}+\beta\left(1-q_{0}\right)\right]\right| \\
& \quad \leq\left|I_{\left\{Y_{n}=0\right\}}-q_{0}\right|+\left|\xi_{0, n}^{(0)}+\sum_{i=2}^{n-1} \xi_{i, n}^{(0)}-\left[\alpha q_{0}+\beta\left(1-q_{0}\right)\right]\right| \\
& \quad \leq 2 .
\end{aligned}
$$

Similarly we get

$$
\left|Z_{n}^{(1)}-Z_{n-1}^{(1)}\right| \leq 2 .
$$

Combining this fact and the martingale property we arrive at

$$
\begin{aligned}
& E\left|S_{t-1}^{(s)}-E\left[S_{t-1}^{(s)}\right]\right|^{2} \\
& =E\left[\sum_{n=1}^{t-1}\left(Z_{n}^{(s)}-Z_{n-1}^{(s)}\right)\right]^{2} \\
& =\sum_{n=1}^{t-1} E\left(Z_{n}^{(s)}-Z_{n-1}^{(s)}\right)^{2} \leq 4 t .
\end{aligned}
$$


Applying Hölder's inequality again, we can get assertion (ii). Thus we complete the proof of Lemma 13.

\section{Conflict of Interests}

The author declares that there is no conflict of interests regarding the publication of this paper.

\section{Acknowledgments}

The author also thanks the anonymous reviewers for valuable comments and suggestions that improved the presentation of this paper. This work is supported by the National Natural Science Foundation of China no. 11201344.

\section{References}

[1] B. Bollobas, Random Graphs, Academic Press, London, UK, 1985.

[2] A.-L. Barabási and R. Albert, "Emergence of scaling in random networks," Science, vol. 286, no. 5439, pp. 509-512, 1999.

[3] B. Bollobás, O. Riordan, J. Spencer, and G. Tusnády, “The degree sequence of a scale-free random graph process," Random Structures and Algorithms, vol. 18, no. 3, pp. 279-290, 2001.

[4] B. Söderberg, "General formalism for inhomogeneous random graphs," Physical Review E: Statistical, Nonlinear, and Soft Matter Physics, vol. 66, no. 6, Article ID 066121, 2002.

[5] B. Bollobás, S. Janson, and O. Riordan, "The phase transition in inhomogeneous random graphs," Random Structures and Algorithms, vol. 31, no. 1, pp. 3-122, 2007.

[6] D. S. Callaway, J. E. Hopcroft, J. M. Kleinberg, M. E. J. Newman, and S. H. Strogatz, "Are randomly grown graphs really random?" Physical Review E: Statistical, Nonlinear, and Soft Matter Physics, vol. 64, no. 2, 2001.

[7] T. S. Turova, "Dynamical random graphs with memory," Physical Review E: Statistical, Nonlinear, and Soft Matter Physics, vol. 70, no. 5, Article ID 059902, 2004.

[8] T. S. Turova, "Long paths and cycles in dynamical graphs," Journal of Statistical Physics, vol. 110, no. 1-2, pp. 385-417, 2003.

[9] T. S. Turova, "Continuity of the percolation threshold in randomly grown graphs," Electronic Journal of Probability, vol. 12, no. 36, pp. 1036-1047, 2007.

[10] T. S. Turova, "Phase transitions in dynamical random graphs," Journal of Statistical Physics, vol. 123, no. 5, pp. 1007-1032, 2006.

[11] R. van der Hofstad, "Critical behavior in inhomogeneous random graphs," Random Structures and Algorithms, vol. 42, no. 4, pp. 480-508, 2013.

[12] R. van der Hofstad, 2013, Random Graphs and Complex Networks, preprint.

[13] F. Papadopoulos, M. Kitsak, M. A. Serrano, M. Boguna, and D. Krioukov, "Popularity versus similarity in growing networks," Nature, vol. 489, pp. 537-540, 2012.

[14] B. Shen, Z. D. Wang, and X. H. Liu, "Sampled-data synchronization control of dynamical networks with stochastic sampling," IEEE Transactions on Automatic Control, vol. 57, no. 10, pp. 2644-2650, 2012.

[15] F. Chung and L. Lu, Complex Graphs and Networks, CBMS regional Conference series in mathematics, 2006. 


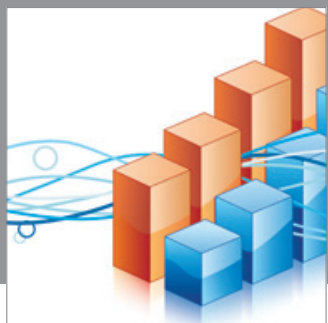

Advances in

Operations Research

mansans

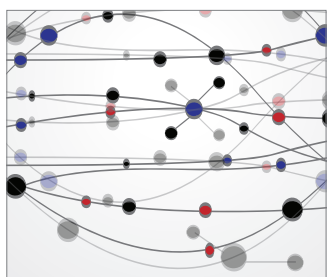

The Scientific World Journal
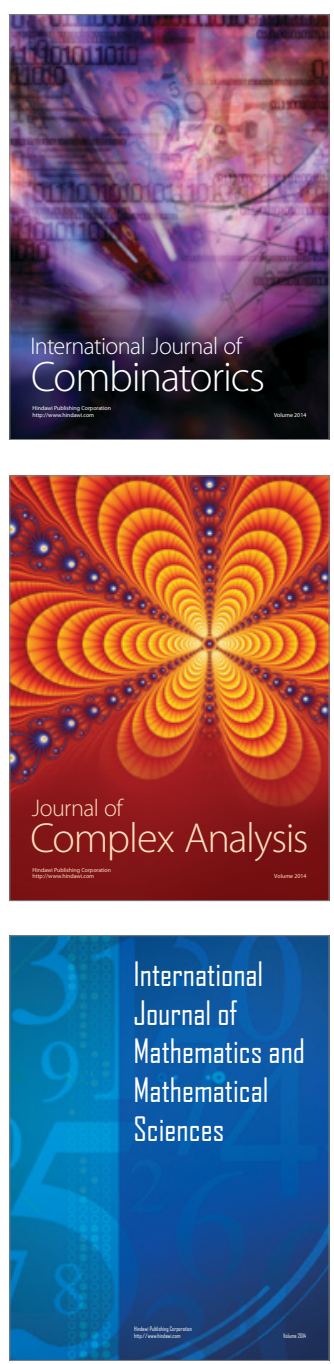
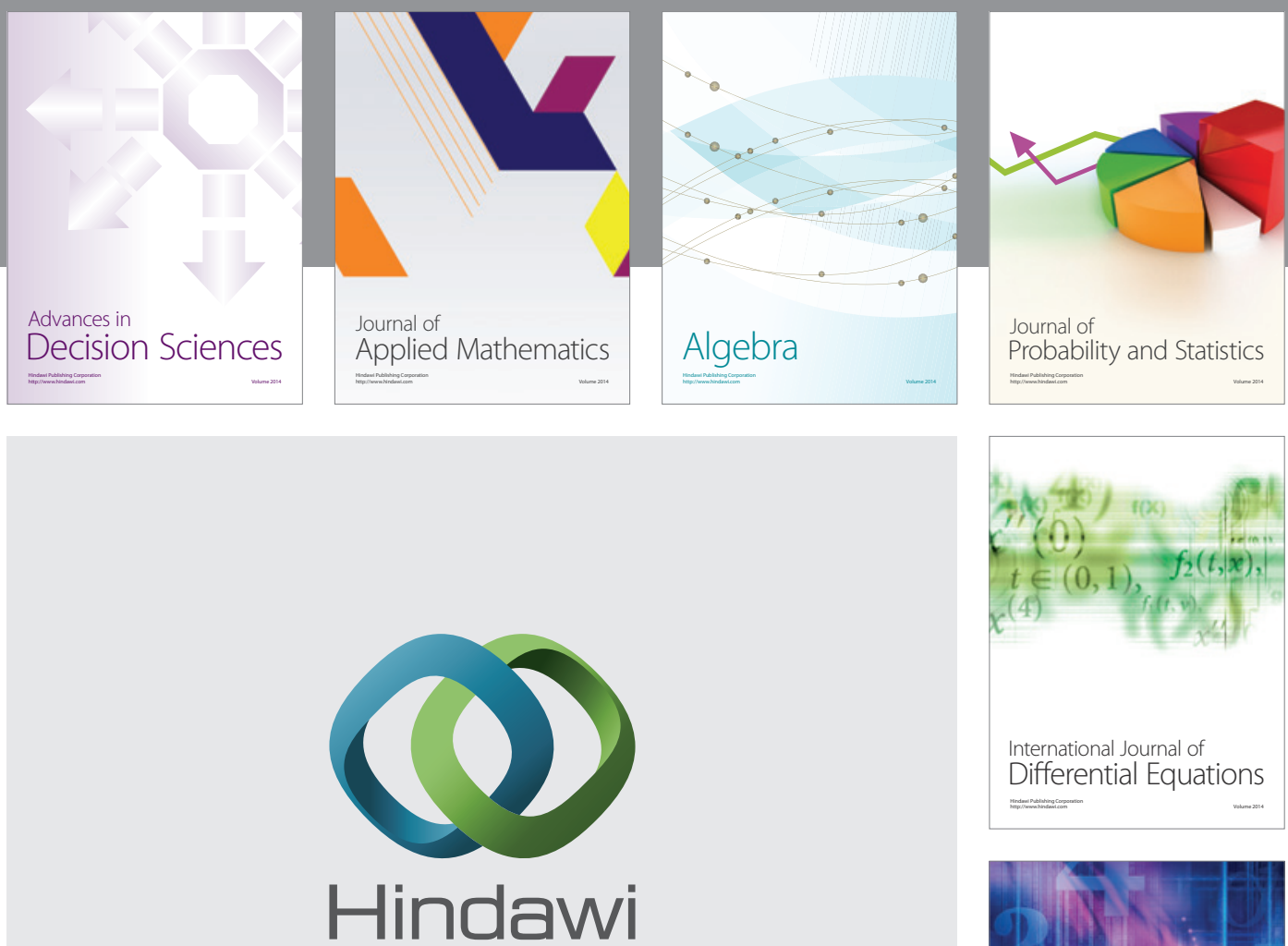

Submit your manuscripts at http://www.hindawi.com
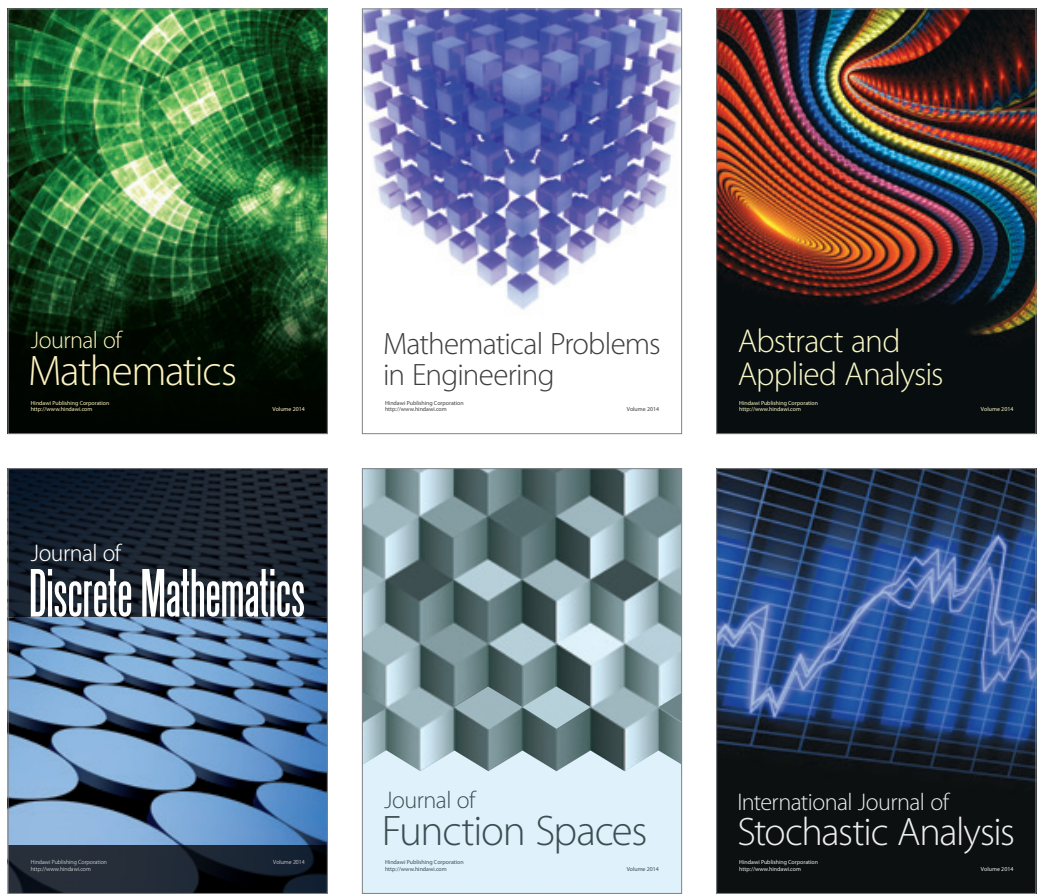

Journal of

Function Spaces

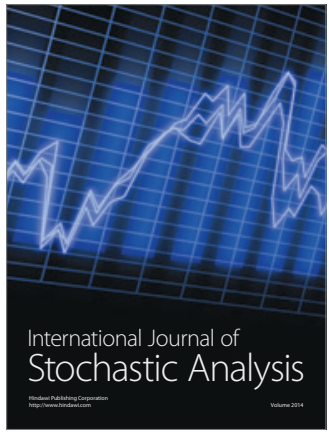

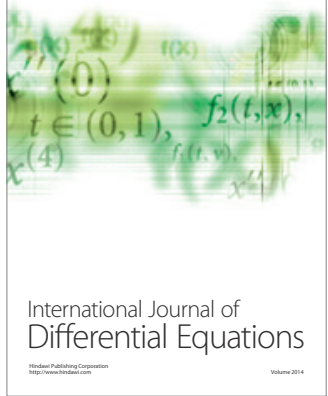
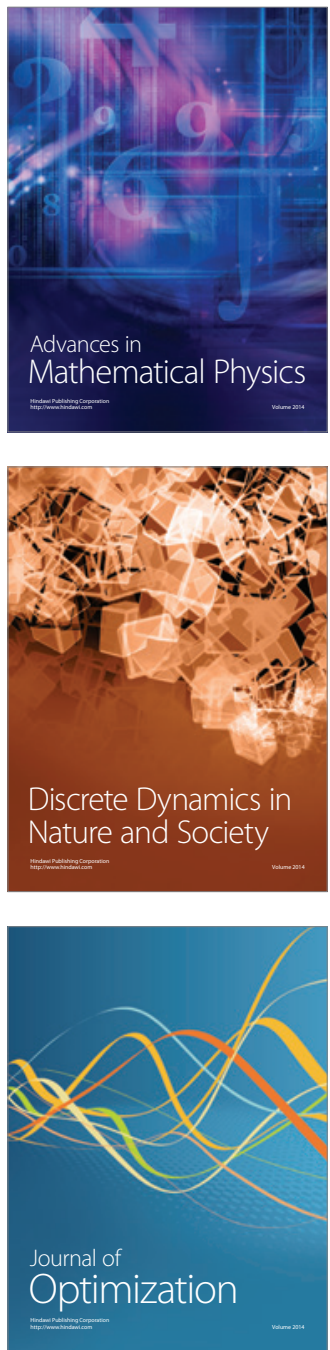